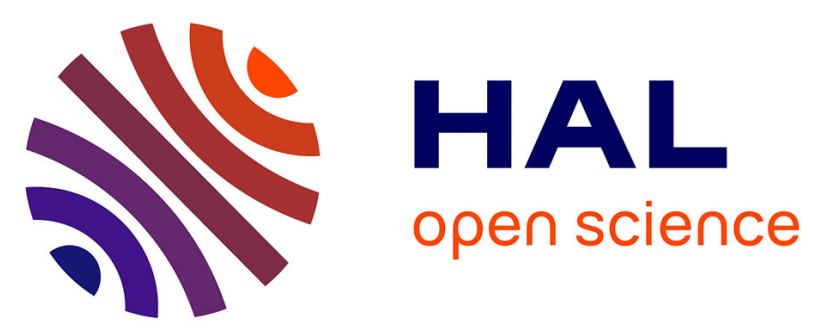

\title{
On the Accuracy of Elastic Strain Field Measurements by Laue Microdiffraction and High-Resolution EBSD: a Cross-Validation Experiment
}

Emeric Plancher, Johann Petit, Claire Maurice, Véronique Favier, Lucie Saintoyant, Dominique Loisnard, N. Rupin, Jean Baptiste Marijon, Olivier Ulrich, Michel Bornert, et al.

\section{To cite this version:}

Emeric Plancher, Johann Petit, Claire Maurice, Véronique Favier, Lucie Saintoyant, et al.. On the Accuracy of Elastic Strain Field Measurements by Laue Microdiffraction and High-Resolution EBSD: a Cross-Validation Experiment. Experimental Mechanics, 2016, 56 (3), pp.483-492. 10.1007/s11340015-0114-1 . hal-02141119

\author{
HAL Id: hal-02141119 \\ https://hal.science/hal-02141119
}

Submitted on 27 May 2019

HAL is a multi-disciplinary open access archive for the deposit and dissemination of scientific research documents, whether they are published or not. The documents may come from teaching and research institutions in France or abroad, or from public or private research centers.
L'archive ouverte pluridisciplinaire HAL, est destinée au dépôt et à la diffusion de documents scientifiques de niveau recherche, publiés ou non, émanant des établissements d'enseignement et de recherche français ou étrangers, des laboratoires publics ou privés. 


\title{
On the Accuracy of Elastic Strain Field Measurements by Laue Microdiffraction and High-Resolution EBSD: a Cross-Validation Experiment
}

\author{
E. Plancher ${ }^{1,2,3}$ - J. Petit ${ }^{4}$. C. Maurice ${ }^{2}$ V. Favier ${ }^{1}$ - L. Saintoyant ${ }^{3}$. D. Loisnard ${ }^{3}$. \\ N. Rupin ${ }^{3}$ - J.-B. Marijon ${ }^{1}$ - O. Ulrich ${ }^{5,6}$ • M. Bornert ${ }^{7}$ - J.-S. Micha ${ }^{5,8}$. \\ O. Robach ${ }^{5,6}$. O. Castelnau ${ }^{1}$
}

\begin{abstract}
Determining the accuracy of elastic strain measurements in plastically deformed alloys is an experimental challenge. To develop a novel cross-validation procedure, a controlled elasto-plastic strain gradient was created in a stainless steel single crystal by four point bending deformation. The corresponding elastic strain field was probed, with an intragranular spatial resolution, in-situ by Laue microdiffraction and ex-situ by High Resolution EBSD. Good agreement is found for the two independent measure-
\end{abstract}

E. Plancher

emeric.plancher@mines-saint-etienne.org

1 Arts et Métiers ParisTech, Laboratoire PIMM, UMR CNRS 8006, 151 boulevard de l'Hôpital, 75013 Paris, France

2 Mines Saint-Étienne, Laboratoire Georges Friedel, UMR CNRS 5307, 158 cours Fauriel, 42100 Saint-Étienne, France

3 EDF, Département MMC, Avenue des renardières, 77818 Moret-sur-Loing, France

4 Université Paris Ouest, Laboratoire LEME, EA4416, 50 rue de Sèvres, 92410 Ville d'Avray, France

5 CEA-CNRS CRG-IF BM32 beamline at ESRF, 6 rue Jules Horowitz, Cedex 9, 38043 Grenoble, France

6 Université Grenoble Alpes, INAC-SP2M and CEA, INAC-SP2M, NRS, 38000 Grenoble, France

7 Université Paris-Est, Ecole des Ponts ParisTech, Laboratoire Navier, 77455 Marne-la-Vallée, France

8 Université Grenoble Alpes, INAC-SPrAM and CNRS, SPrAM and CEA, INAC-SPrAM, PCI, 38000 Grenoble, France ments and the predictions of a mechanical model, at plastic strains below $0.5 \%$. The accuracy of the measurements is estimated at $3.2 \times 10^{-4}$.

Keywords Synchrotron radiation · HR-EBSD · Laue microdiffraction · Austenitic steel $\cdot$ Stainless steel · Laue-DIC

\section{Introduction}

Diffraction-based techniques probing elastic strain at the micron scale are promising tools to investigate the mechanical behaviour of polycrystals. In plastically deformed materials, elastic strain fields (or associated stress fields) always develop heterogeneously at the grain level, due to the anisotropic mechanical behavior. Damage nucleation occurs in highly stressed and strained locations. Therefore spatial quantification of strain heterogeneities is of great importance to predict weak spots in the microstructure.

Among the available techniques, X-ray Laue microdiffraction [1] and high angular resolution electron backscatter diffraction (HR-EBSD) [2] are particularly attractive. They allow for probing 2D fields with a submicrometer step size and measure at least the full deviatoric part of the elastic strain tensor. These techniques are very sensitive to strain variations : $2.10^{-4}$ for HR-EBSD and down to $10^{-5}$ for Laue microdiffraction with the relative procedure called Laue-DIC [3, 4].

Accuracy assessment of the two techniques has been carried out mostly on flat semi-conductors [2, 4-7] and on an annealed steel sample [8], in the elastic regime. In these favourable conditions, the accuracy of HR-EBSD 
and Laue microdiffraction respectively attains their ultimate sensitivity.

However, in plastically strained metals measuring and validating local elastic strains is much more difficult. Although there have been several publications describing apparent strain measurements by these local diffraction techniques, some values reported are surprising given the macroscopic mechanical characteristics of the specimen. For example, in copper samples with a macroscopic yield stress below $70 \mathrm{MPa}$, regions in which local stress levels exceed $500 \mathrm{MPa}$ were reported from Laue microdiffraction [9] and HR-EBSD experiments [10]. It is unclear whether the crystal at the local scale can actually withstand residual stresses up to several times the macroscopic yield limit. As suggested by [11] for the observations of [9] and other examples, large strain values may originate from experimental and data processing artefacts rather than being relevant for a true mechanical state. Artefacts are known to create non-physical strains also known as spurious deformation [8] or phantom strains [12].

As outlined below, there are many additional difficulties when measuring local strains in plastically deformed metals, but one of the major stumbling blocks is the lack of obvious validation procedures. Validation is a critical issue but to our knowledge, it has not been attempted previously on a material deformed elasto-plastically. The basic reason is that there are no standard samples with well-known elasto-plastic strain fields. This work aims to compare two methods of strain field analysis on a relatively simple elasto-plastic deformation state with the goal of proposing a cross-validation procedure.

Artifacts of strain measurement in plastically deformed metals stem from (i) the presence of orientation and rotation gradients, (ii) the absence of clear reference strain states and (iii) poorly controlled experimental conditions such as sample misalignment and set-up calibration.

Rotation gradients formed during plastic deformation are an issue for the standard cross-correlation procedure in HR-EBSD [12, 13]. Additional image processing of the diffraction patterns (so-called "remapping") is needed. Unfortunately remapping is highly dependent on the actual geometry of the diffraction setup, which is not known to a satisfactory precision [14-16]. Rotation gradients create curvy and elongated spots in Laue microdiffraction patterns. Defining spot position and displacement can become difficult. Deconvolution of the diffraction signal according to the depth of material is necessary to retrieve circular spots [17].

Experimental artefacts encompass issues with the geometry of the diffraction experiment. Calibration of the set-up is a critical step in Laue microdiffraction $[6,8,18]$ and HR-
EBSD [16]. In the scanning electron microscope, suitable scanning conditions should be achieved for an accurate evaluation of the strain field [19]. Surface topography of the polished sample is also suspected to have an influence on the measurement [20] as the HR-EBSD procedure usually requires a perfectly flat surface.

The presence of artefacts being highly probable, elastic strain fields used to study plastic behavior should be validated. As a first step forward from the ideal elastic case, this work explores elastic strain fields developing at the elasto-plastic transition of a steel sample. Our aim is to evaluate the accuracy of Laue microdiffraction and HR-EBSD measurements in the low plastic regime.

A stainless steel single crystal is deformed in-situ with a bending device and elastic strains are monitored by LaueDIC. The observations clearly show the transition from pure elastic behavior to elasto-plastic behavior. Measured elastic strains are then compared with the prediction of a mechanical model, using the known characteristics of the material. Once the specimen is unloaded, residual elastic strains are measured by Laue-DIC and HR-EBSD. An estimation of the accuracy of elastic strain measurements in the plastic domain is proposed.

\section{Material and Methods}

\section{Material and Specimen Preparation}

The stainless steel single crystal was grown by directional solidification, in a horizontal furnace under argon pressure. Its chemical composition is close to that of a $316 \mathrm{~L}$ stainless steel : Fe-17.0Cr-14.6Ni-2.3Mo (wt\%). The crystal is carbon- and nitrogen-free.

Sample preparation is a critical issue for HR-EBSD and Laue microdiffraction. The sample was cut by spark erosion to an initial shape of $30 \times 5 \times 0.8 \mathrm{~mm}^{3}$, with $<100>$ crystal axes aligned with the sample axes $\left( \pm 3^{\circ}\right)$. The two $30 \times$ $5.0 \mathrm{~mm}^{2}$ faces were mechanically polished with hard cloths down to $1 \mu \mathrm{m}$ diamond grade, removing $150 \mu \mathrm{m}$ of material affected by spark erosion from each side. The observed face was also mechanically polished and then electropolished for $45 \mathrm{~s}$ (at $6{ }^{\circ} \mathrm{C}$ under $30 \mathrm{~V}$ in Struers A2 solution) to eliminate any residual back stresses. The final shape of the sample is $30 \times 4.8 \times 0.50 \mathrm{~mm}^{3}$.

The specimen preparation quality was checked using channelling contrast imaging in a Scanning Electron Microscope (SEM). A uniform contrast was obtained from the observed area, indicating the presence of a nearly perfect single crystal without any local misorientation. The sample surface topography was probed using optical profilometery. 
The specimen surface is planar with irregularities under $500 \mathrm{~nm}$, except for 10 to $30 \mu \mathrm{m}$ close to the edges.

\section{Laue-DIC in-situ Experiment}

The geometry of the bend test is presented in Fig. 1, showing the expected development of strain gradients in the thickness of the sample. Tension and compression are observed simultaneously, delimited by a neutral axis. The latter is considered unstrained and situated at half-width of the sample. Therefore the neutral axis is used for the reference diffraction patterns for Laue-DIC and HR-EBSD relative measurements. Four point bending is convenient because strain distribution between the two inner pegs is uniform along the $y$ axis.

The experiment was performed in-situ on the Laue microdiffraction setup available at the French CEA-CNRS beamline BM32 at ESRF [21, 22]. Loading was incremented regularly at a speed of $0.2 \mathrm{~mm} / \mathrm{min}$, according to the force-displacement curve presented in Fig. 1. The bending pegs position was kept constant during diffraction acquisitions. $F$ sometimes drops due to visco-plastic relaxation processes when loading is interrupted. When relaxation has terminated and the applied force is stabilized (about $30 \mathrm{~min}$ after loading interruption), elastic strains are measured on a profile across the thickness of the sample, along the $x$ axis. Nine levels of loading are investigated, including four before plastification and one after unloading.

Laue diffraction patterns were acquired using a Photonic Science VHR X-ray detector $(2774 \times 2594$ pixels, $31 \mu \mathrm{m}$ pixel size), positioned $60 \mathrm{~mm}$ away from the sample. The white X-ray beam is composed of wavelengths between 5 and $23 \mathrm{keV}$. In pure iron, the penetration depth for $5 \mathrm{keV}$ radiations is $9 \mu \mathrm{m}$. It reaches $77 \mu \mathrm{m}$ at $23 \mathrm{keV}$ [23]. There-
Fig. 1 (a) Configuration of the bending experiment. Characteristic lenghts of the device are $d=8.5 \mathrm{~mm}$, $c=3 \mathrm{~mm}$ and

$l=2 \times(c+d)=23 \mathrm{~mm}$. Thickness of the sample $e=0.5 \mathrm{~mm}$ and its height $b=4.8 \mathrm{~mm}$ along the $Z$ direction are recalled. Insert shows notations used for the mechanical model. (b) Associated force-displacement curve (a)

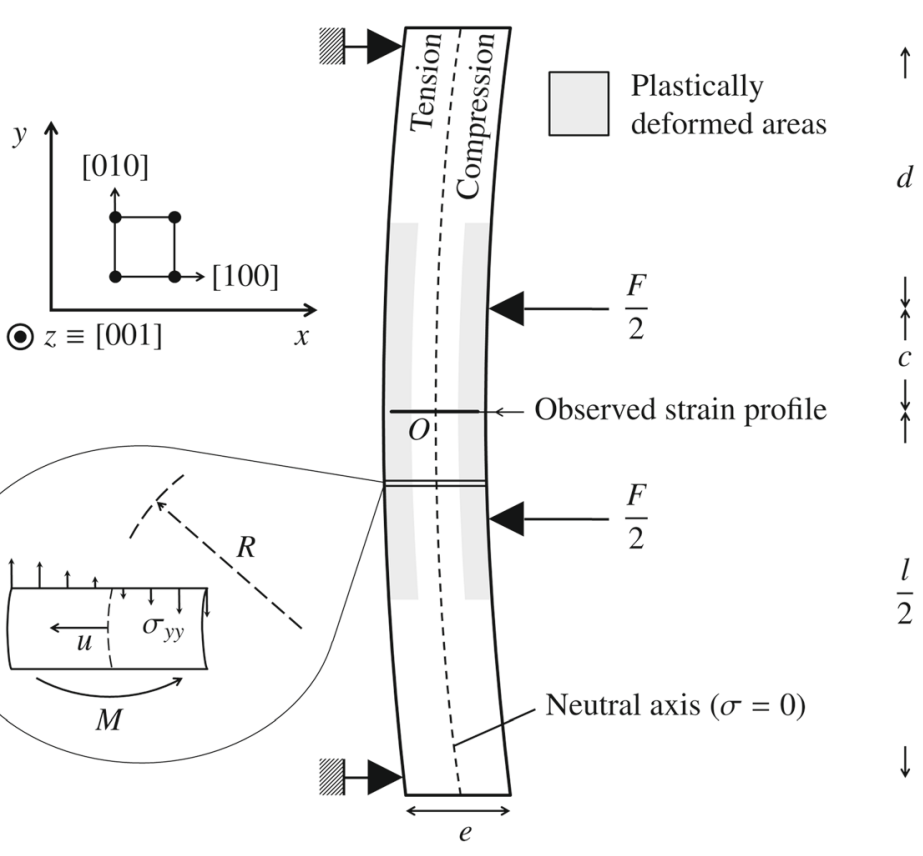

(b)

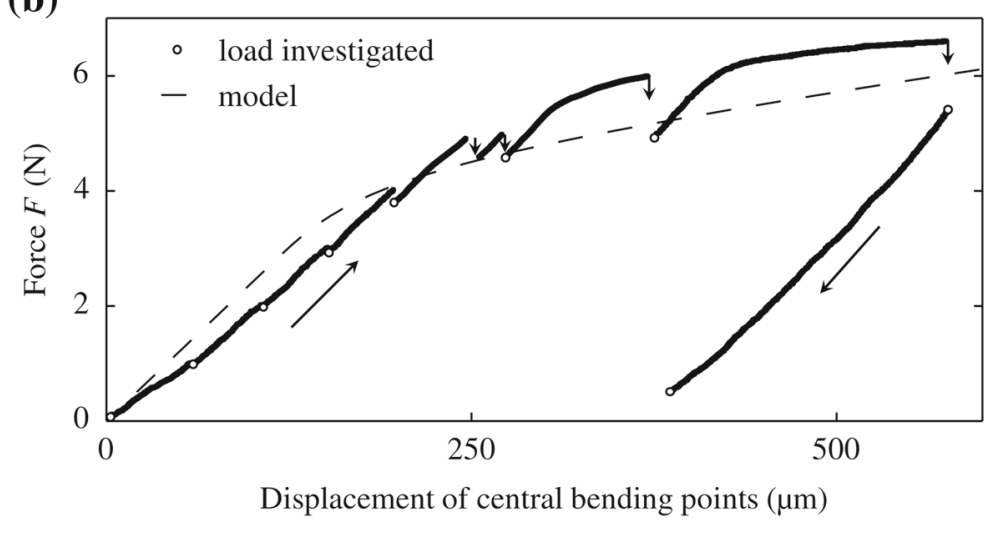


fore the depth of material probed is less than $\sim 40 \mu \mathrm{m}$. The incoming X-ray beam (that lies in the $y z$ plane) is tilted $40^{\circ}$ around the $\mathrm{x}$ direction with respect to the specimen surface. The beam cross-section size was set close to $0.8 \times 0.6 \mu \mathrm{m}^{2}$. Line scans were acquired with a $2 \mu \mathrm{m}$ step-size along the $x$ axis. We used a germanium single crystal to calibrate the diffraction geometry. Regarding data processing, 16 to 18 reflections are used to extract the deviatoric strain in the steel specimen with the Laue-DIC technique [3]. For each reflection, the intensity follows a gaussian distribution around the well defined peak maximum. No significant evolution of the peaks' shape is observed with the loading. The original shape has little influence on Laue-DIC results. Basic local transformation algorithms are used during DIC processing to measure the peak displacements.

\section{HR-EBSD ex-situ Acquisition}

For HR-EBSD analysis, we used a Zeiss Supra 55VP FEGSEM operating at $20 \mathrm{kV}$ with a probe current of $\sim 2.4 \mathrm{nA}$. The penetration depth of backscatter electrons for the steel single-crystal is evaluated by Monte-Carlo simulations [24] (for $600 \mathrm{eV}$ as a maximum energy-loss) at $25 \mathrm{~nm}$. EBSD acquisition with a step-size of $0.1 \mu \mathrm{m}$ was carried out using a HKL system (Oxford Instruments) composed of a
NordlysII camera and the channel 5 software suite. Patterns were recorded at full resolution $(1344 \times 1024$ pixels $)$, using averaging on three frames. Data were post-processed with our own StrainCorrelator software [7, 12].

An experimental challenge was to finely control the position of the observed surface and scanning conditions, in order to obtain a $500 \mu \mathrm{m}$ long profile with limited artefacts. To achieved this purpose, we used a specific sample holder designed to precisely position the observed surface parallel to the stage reference plane. A silicon grid was imaged to calibrate scanning parameters (tilt correction and scan rotation).

\section{Results}

In this experiment, as strain and rotation components barely reach $10^{-3}$, we can use an infinitesimal strain analysis. The deformation gradient tensor $\boldsymbol{F}$ is additively decomposed into small strain $\boldsymbol{\varepsilon}$ and rotation $\omega$ tensors:
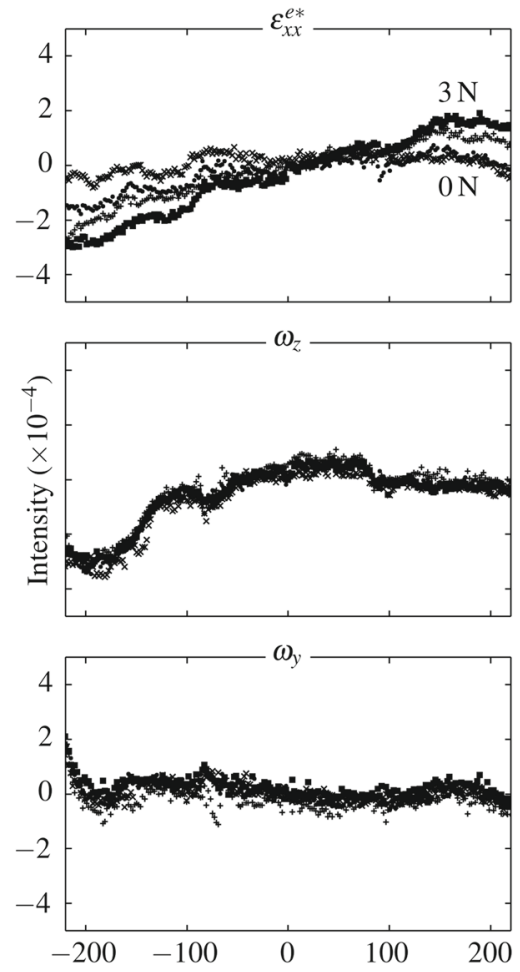
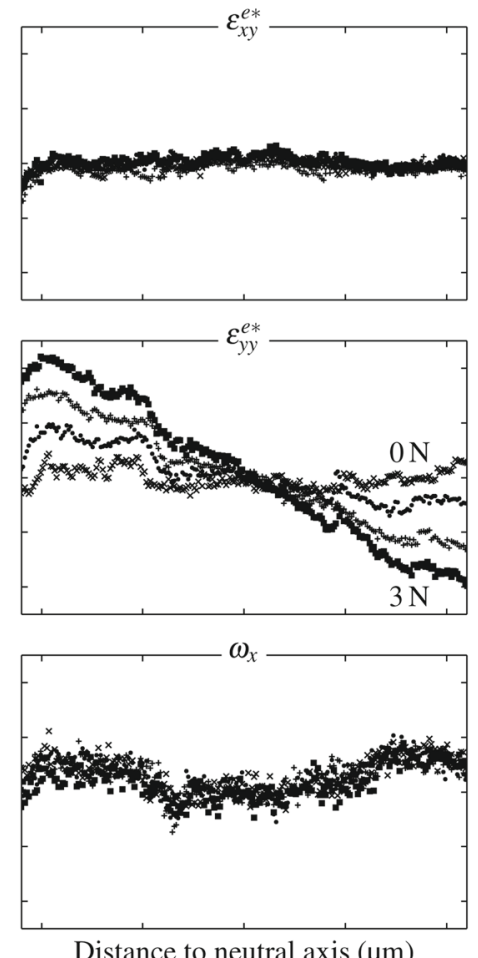

Distance to neutral axis $(\mu \mathrm{m})$
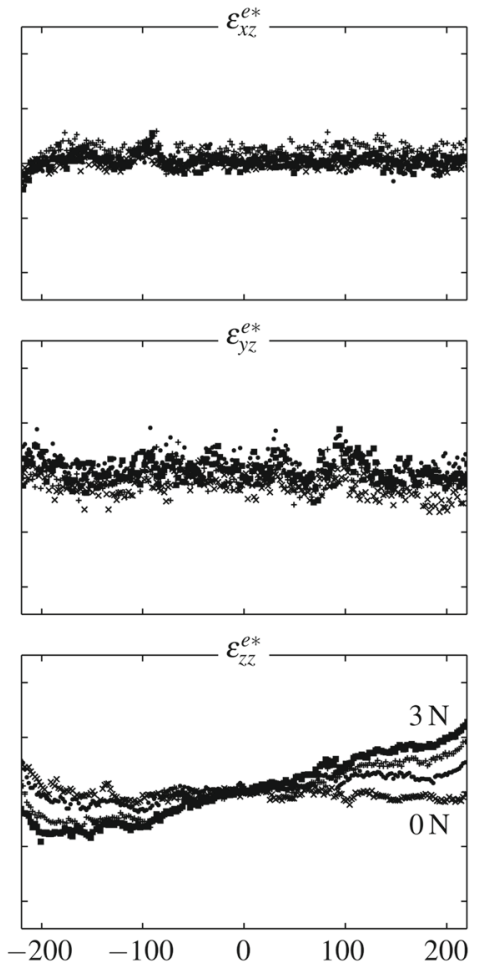

Fig. 2 Deviatoric elastic strain and misorientation on a through-thickness profile along the $x$ axis of the sample, measured by Laue-DIC at 0 N, $1 \mathrm{~N}, 2 \mathrm{~N}$ and $3 \mathrm{~N}$ loads. All results are relative to the reference point taken at half-width of the sample (x direction) and assumed unstrained. A pure elastic behavior of the crystal is observed : a linear strain profile develops on tensile components while shear and rotation components remain identical to the initial ones 

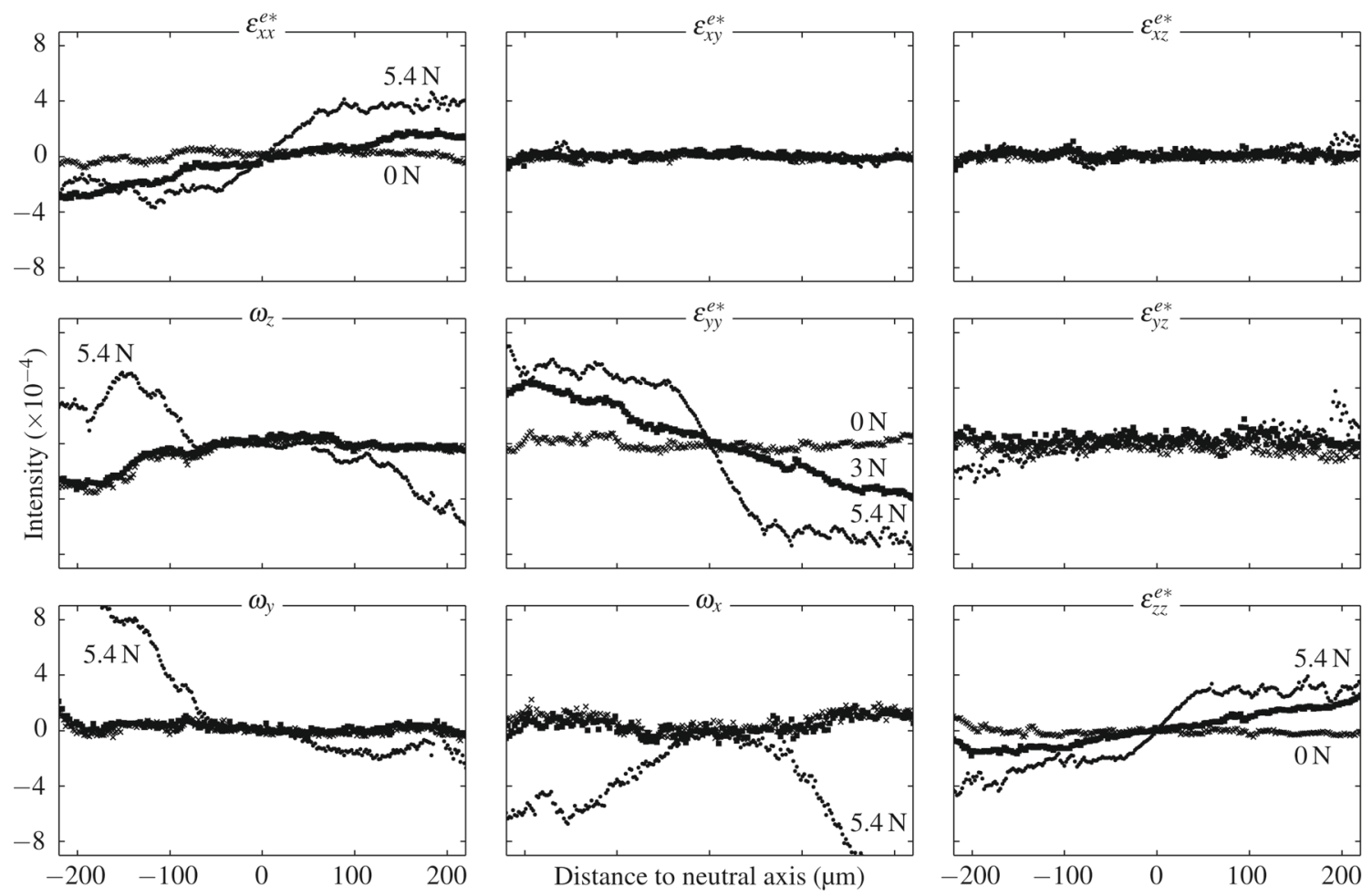

Fig. 3 Deviatoric elastic strain and misorientation on a through-thickness profile along the $x$ axis of the sample, measured by Laue-DIC at 0 N, $3 \mathrm{~N}$ and $5.4 \mathrm{~N}$ loads. All results are relative to the reference point taken at half-width of the sample ( $x$ direction) and assumed unstrained. The elasto-plastic behavior of the crystal is illustrated at $5.4 \mathrm{~N}$ load : a non linear strain profile is present on tensile components. Rotation components have evolved as the result of plastic processes

The strain tensor $\boldsymbol{\varepsilon}$ is then decomposed into elastic and plastic parts, $\boldsymbol{\varepsilon}=\boldsymbol{\varepsilon}^{e}+\boldsymbol{\varepsilon}^{p}$. Hooke's law is written using the stiffness tensor $\boldsymbol{C}$ as $\boldsymbol{\sigma}=\boldsymbol{C}: \boldsymbol{\varepsilon}^{e}$. The experimental results are shown using only the deviatoric part of the elastic strain tensor $\varepsilon^{e *}$, since the used diffraction techniques are insensitive to its hydrostatic part. Owing to the cubic symmetry of the crystal lattice, the deviatoric stress $\sigma^{*}$ can also be computed with $\sigma^{*}=\boldsymbol{C}: \boldsymbol{\varepsilon}^{e *}$ as explained in [3].

Figures 2 and 3 show the evolution of strain and misorientation measured by Laue-DIC on a through-thickness profile along the $x$ axis, at the centre of the sample. Tensile $\left(\varepsilon_{i i}^{e *}\right)$ and shear $\left(\varepsilon_{i j}^{e *}\right)$ strain components are presented with respect to the sample axis introduced in Fig. 1. Rotation components $\left(\omega_{i}\right)$ are expressed in the same system of coordinate. All results are relative to the reference point taken at half-width of the sample ( $\mathrm{x}$ direction), assumed unstrained because situated on the neutral axis.
Before loading (Fig. 2), small elastic strain fluctuations around zero are observed with an amplitude of $10^{-4}$. Misorientations observed in the single crystal are less than $3.10^{-4} \mathrm{rad}$ or $0.02^{\circ}$.

At $1 \mathrm{~N}, 2 \mathrm{~N}$ and $3 \mathrm{~N}$ loads, a linear and symmetric tensile strain profile develops in the sample. The evolution of the profile slopes appears proportional to the load. Component $\varepsilon_{x x}^{e *}$ and $\varepsilon_{z z}^{e *}$ are negative on the left side of the neutral axis while $\varepsilon_{y y}^{* *}$ is positive. An opposite behaviour is observed on the right side. Fluctuations around the linear tendency are observed. These fluctuations are similar in shape with the initial ones. They essentially correspond to the initial strain state of the specimen rather than being a consequence of the loading. Shear and rotation components stay identical to their initial values. An evaluation of the dispersion for these components is presented in Table 1. The dispersion is higher on shear component $\varepsilon_{y z}^{e *}$ and $\varepsilon_{x z}^{e *}$ and associated
Table 1 Dispersion of Laue-DIC shear and rotations components around their mean profile during elastic loading

\begin{tabular}{lcccccc}
\hline & $\varepsilon_{x y}^{e *}$ & $\varepsilon_{y z}^{e *}$ & $\varepsilon_{x z}^{e *}$ & $\omega_{x}$ & $\omega_{y}$ & $\omega_{z}$ \\
\hline Standard deviation from mean profile $\left(\times 10^{-4}\right)$ & 0.11 & 0.27 & 0.17 & 0.20 & 0.17 & 0.13 \\
\hline
\end{tabular}



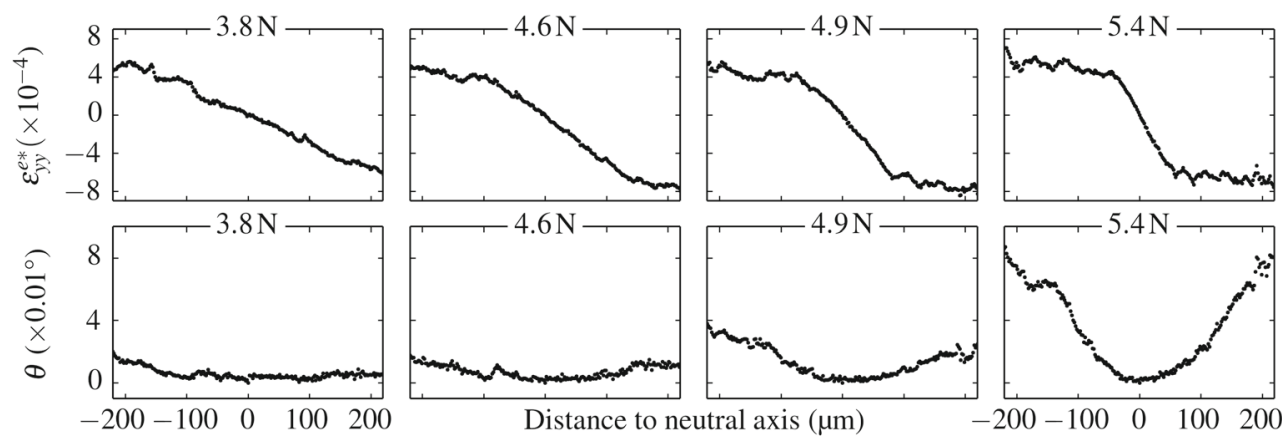

Fig. 4 Evolution of the elastic strain component $\varepsilon_{y y}^{e *}$ and misorientation angle $\theta$ during deformation in the elasto-plastic transition. All results are relative to the reference point taken at half-width of the sample ( $x$ direction) and assumed unstrained. Development of a non-linear elastic strain profile is observed as plastic deformation associated with crystal rotations develops from the sides of the sample

rotation component $\omega_{x}$ and $\omega_{y}$ (due to measurement noise and limited pole figure coverage [8]) than for $\varepsilon_{x y}^{e *}$ and $\omega_{z}$.

At $5.4 \mathrm{~N}$ the tensile strain profile is composed of three segments as illustrated in Fig. 3. On the left and right sides, the slopes are low but become steep close to the neutral axis. Misorientation distribution is different from the initial one in the regions where the slopes are low. Limited evolution of the shear component $\left(<4.10^{-4}\right)$ is detected in the $\varepsilon_{y z}^{e *}$ component.

For clarity purposes, profiles acquired at intermediary loads ( $3.8 \mathrm{~N}, 4.6 \mathrm{~N}$ and $4.9 \mathrm{~N}$ ) are presented through the evolution of the $\varepsilon_{y y}^{e *}$ component and misorientation angle $\theta$. The misorientation angle $\theta$ is the angle of the rotation described by the $\omega$ tensor. As seen in Fig. 4, at $3.8 \mathrm{~N}$ the three segment profile is observed with low slope regions limited to the very side of the sample. At $4.6 \mathrm{~N}$ and $4.9 \mathrm{~N}$ the low slope regions extend gradually toward the neutral axis. Rotations components show a similar evolution, as demontrated by the evolution of the misorientation angle. Areas where rotations components are different from the initial ones evolve from the side toward the neutral axis. Their extent is always identical to the one of the low slope regions of the tensile profile.

Figure 5 shows the compression side of the fully bent crystal, as visualized by electron channeling contrast after unloading. Markings consistent with slip on $\{111\}$ planes are observed in the plastified region whereas none are present in the purely elastically strained region, near the neutral axis.
After unloading, the previous localized plasticity gives rise to residual elastic strains (cf. Fig. 6). The three observed regions in the profile correspond to the two elasto-plastic and one elastic zones. Maximum values measured for the $\varepsilon_{y y}^{e *}$ component are of order $4 \times 10^{-4}$.

HR-EBSD measurements of the residual strain state are also presented in Fig. 6. Thanks to the small step size, clear trends are observed despite the dispersion of the data. To quantify the dispersion, the trend in each component is estimated with a moving average procedure over 300 points. Standard deviation of the distance between data points and the average trend is equal or smaller than $10^{-4}$.

General good agreement is found between the average profile from Laue-DIC and HR-EBSD of the same residual strain state. Table 2 regroups the mean values of the distance between the average trend of HR-EBSD and Laue-DIC data points, calculated in the plastically deformed regions only. The mean distance between profiles is smaller than $3.2 \times 10^{-4}$.

\section{Discussion}

\section{Elasto-Plastic Transition Observed by Laue-DIC}

Initially the crystal is nearly perfect (i.e. no sub-grain) and stress free as expected. Up to $3 \mathrm{~N}$, the sample response is purely elastic. At the macroscopic level, the forcedisplacement curve (cf. Fig. 1(b)) show a linear relationship

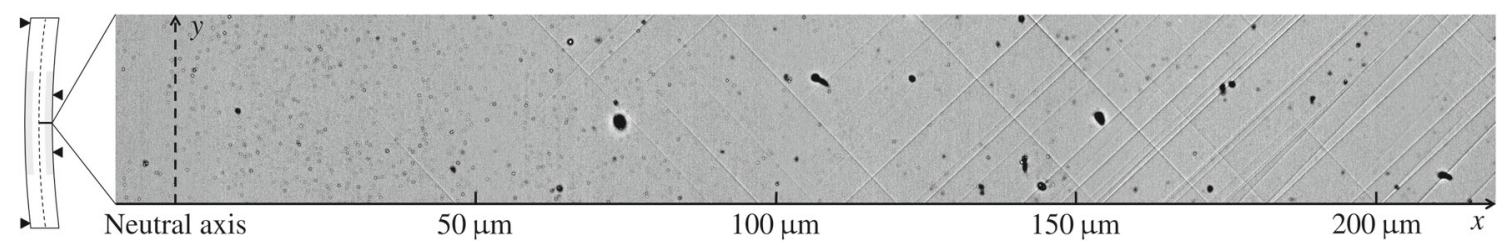

Fig. 5 Backscattered electron channelling contrast observation of slip markings near the surface of the fully bent single crystal after unloading 

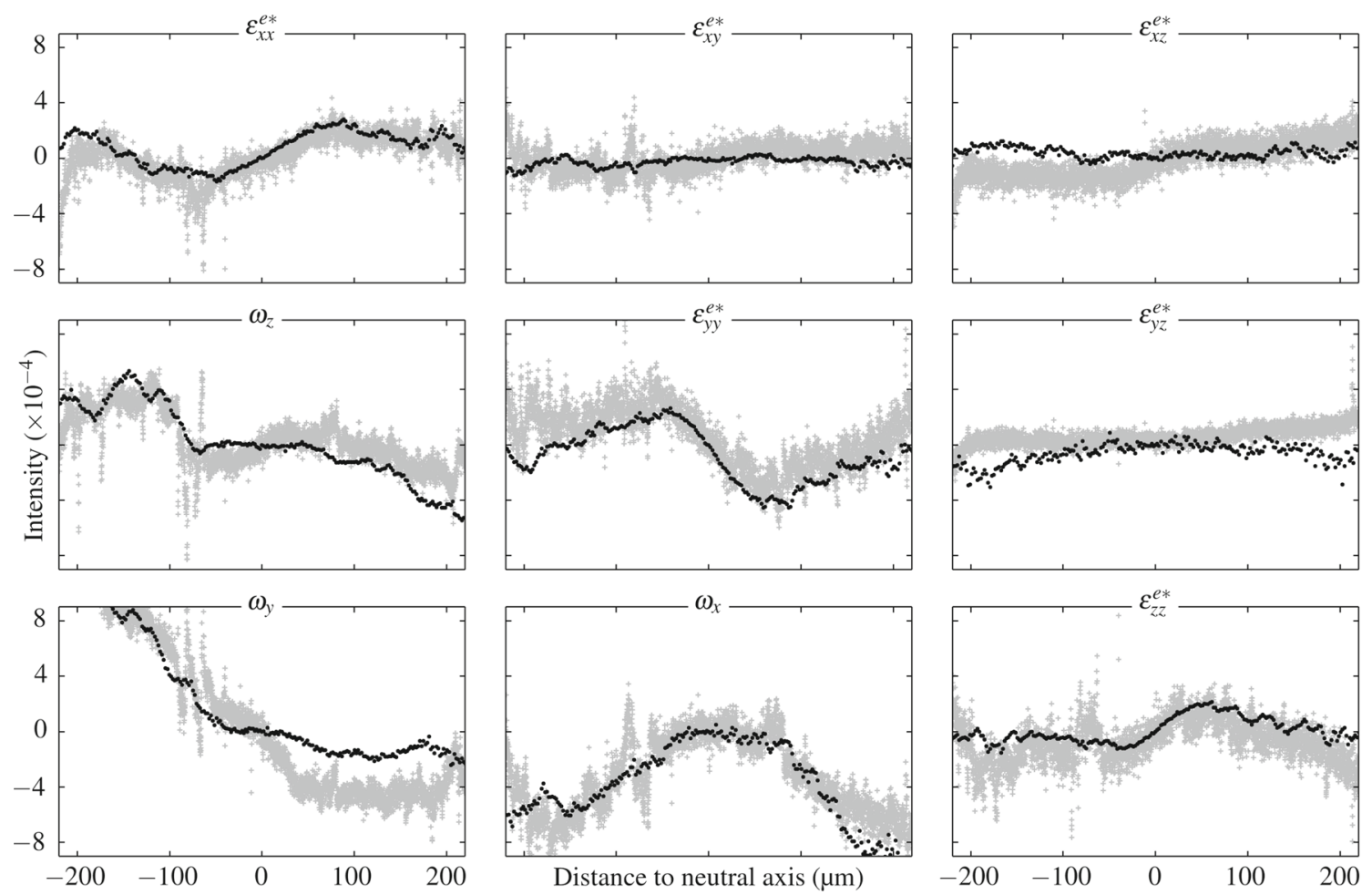

Fig. 6 Residual deviatoric elastic strain and misorientation on the same through-thickness profile along the $x$ axis of the sample, measured by Laue-DIC (black dots) and HR-EBSD (grey dots). All results are relative to the reference point taken at half-width of the sample ( $x$ direction) and assumed unstrained

with no drop when loading is interrupted. At the local scale, a linear tensile strain state is measured. The repartition of the strain between $\varepsilon_{x x}^{e *}, \varepsilon_{y y}^{e *}$ and $\varepsilon_{z z}^{e *}$ is consistent with the Poisson effect associated with a uniaxial stress state along the $\mathrm{y}(<010>)$ axis. Here the specific specimen orientation indicated in Fig. 1 was chosen so that the symmetry of the loading matches the symmetry of the crystal lattice. One therefore expect $\varepsilon_{x x}^{e *}=\varepsilon_{z z}^{e *}$ with all shear components vanishing [25]. The elastic strain deformation is proportional to the distance from the neutral axis as expected from Bernoulli standard beam theory. No shear strain is observed meaning that pure bending is achieved at the location of the profiles. The absence of crystal rotation is also a clue that deformation is purely elastic.

At $3.8 \mathrm{~N}$, the stress state reaches the yield limit on the sides of the sample (i.e. far from the neutral axis). Plastic deformation occurs and the elastic strain tends to saturate, introducing two breaks in the linear profile. Tensile plastic strain is found on the left side of the profile whereas plastic compression occupies the right side. As the load is increased, the slope near the neutral axis becomes steeper. More volume attains the yield limit and the plastic areas extend toward the neutral axis. As plastic deformation increases on the sample sides, hardening occurs explaining non-zero slopes in the plastically deformed regions. A sign of plastic deformation is the evolution of the rotation components as a consequence of dislocation glide and accumulation in the form of GNDs. Small evolution of the shear components may be a consequence that after some plasticity the material behaviour become heterogeneous in the sample. Therefore pure bending conditions are not respected anymore at the local scale.

This interpretation of strain measurements is consistent with the arrangement of slip markings. Close to the neutral axis, no slip occurs as the deformation stays elastic. In Fig. 5, slip markings are detected between $40 \mu \mathrm{m}$ and $75 \mu \mathrm{m}$ away from the neutral axis in agreement with the transition observed in the strain profile. The number of slip marking increase with the distance to the neutral axis, underlying an expected increase in plastic deformation.
Table 2 Mean distance $\left(\times 10^{-4}\right)$ between Laue-DIC data and HR-EBSD average trend in plastically deformed areas

\begin{tabular}{lccccccccc}
\hline & $\varepsilon_{x x}^{e *}$ & $\varepsilon_{x y}^{e *}$ & $\varepsilon_{y y}^{e *}$ & $\varepsilon_{y z}^{e *}$ & $\varepsilon_{x z}^{e *}$ & $\varepsilon_{z z}^{e *}$ & $\omega_{x}$ & $\omega_{y}$ & $\omega_{z}$ \\
\hline Distance $\left(\times 10^{-4}\right)$ & 1.48 & 1.48 & 3.18 & 3.00 & 2.61 & 2.14 & 2.52 & 3.86 & 2.72 \\
\hline
\end{tabular}


The elasto-plastic strain profile was manufactured in controlled conditions to test the validity of elastic strain measurements in the plastic region. By design, the experiment is convenient for mechanical modelling. Indeed, initially the material is proven to be homogeneous and strain-free. Moreover a pure bending loading is achieved at the centre of the sample. Therefore only the plastic constitutive law of the sample is a real unknown. In the following we will verify whether the measured strain profiles fit with a mechanical model using an elasto-plastic constitutive law.

\section{Measurement Validation with Mechanical Modelling}

To compare with experimental results, we build an analytical model based on classical beam theory. This 1D model is able to predict only the evolution of tensile strain components $\varepsilon_{y y}$ and $\sigma_{y y}$. Rotation and shear components are not considered.

A bilinear constitutive law is used to describe the elastoplastic mechanical behaviour. The yield limit $\sigma_{0}$ is reached for $\varepsilon_{y y}=\varepsilon_{0}$. Hardening is governed by a hardening modulus $H$,

$\sigma_{y y}=\left\{\begin{array}{ll}-\sigma_{0}+H\left(\varepsilon_{y y}-\varepsilon_{y y}^{e}\right) & \text { if } \varepsilon_{y y}<-\varepsilon_{0} \\ E \varepsilon_{y y} & \text { otherwise } \\ \sigma_{0}+H\left(\varepsilon_{y y}-\varepsilon_{y y}^{e}\right) & \text { if } \varepsilon_{y y}>\varepsilon_{0}\end{array}\right.$.

The Young modulus $E$ in equation (1) is computed from the crystal elasticity constants. Cubic symmetry is considered for the stiffness tensor associated with the austenitic FCC single crystal. As the sample is loaded along $<100>$ crystallographic directions,

$E=E_{<100>}=C_{11}\left(\frac{1-3 v^{2}-2 v^{3}}{1-v^{2}}\right) \quad$ with $\quad v=\frac{1}{1+\frac{C_{11}}{C_{12}}}$.

Here only the parameters $\sigma_{0}$ and $H$ associated with the plastic flow are true unknown.

Pure bending conditions are assumed. The bending moment $M$ and the bending radius $R$ are defined along the neutral axis of the sample (cf. Fig. 1). Following Bernoulli hypotheses, displacements $\underline{\boldsymbol{u}}$ are carried by the $x$ axis $(\underline{\boldsymbol{u}}=-u(y) \underline{\boldsymbol{x}})$. Only tensile components $\varepsilon_{y y}$ and $\sigma_{y y}$ are considered:

$\varepsilon_{y y}=-x \frac{1}{R(y)}$.

Denoting $b$ the height of the beam $(b=4.8 \mathrm{~mm})$ by definition,

$\frac{M(y)}{b}=-\int_{-\frac{e}{2}}^{\frac{e}{2}} x \sigma_{y y} \mathrm{~d} x$.
Using equations (3) and (1), one can solve equation (4) to obtain an analytical expression for the bending moment $M$ as a function of $R$ :

$\frac{M}{b}=\frac{E}{H+E}\left[\frac{1}{12} H e^{3} \frac{1}{R}+\frac{1}{4} \sigma_{0} e^{2}-\frac{1}{3} \frac{\sigma_{0}^{3}}{E^{2}} R^{2}\right]$.

Equation (5) can be inverted to obtain the function $R=$ $g(M)$. Global equilibrium of every slice of matter gives the relation between $F$ and $M$,

$M(y)=\frac{F}{2} \times \begin{cases}\left(\frac{l}{2}-y\right) & \text { if } y>\frac{l}{2}-d \\ d & \text { otherwise } \\ \left(-\frac{l}{2}-y\right) & \text { if } y<-\frac{l}{2}+d\end{cases}$

where $d$ and $l$ are defined in Fig. 1 .

Given a loading force $F$ and a set of parameters $\left(\sigma_{0}, H\right)$, we use $g$ to compute $R$ along the neutral axis. With a combination of equations ( 3 ) and (1), the profile of $\sigma_{y y}$ across the sample can be evaluated at the locations of the experimental data. The deviatoric tensile component $\varepsilon_{y y}^{e *}$ of the elastic strain tensor is then calculated as

$\varepsilon_{y y}^{e *}=\frac{2}{3}(1+v) \varepsilon_{y y}^{e} \quad$ with $\quad \varepsilon_{y y}^{e}=\frac{1}{E} \sigma_{y y}$.

The displacement of the bending pegs, knowing $R(y)$ at a loading step, can be retrieved by integration of the following fundamental equation of standard beam theory :

$\frac{1}{R(y)}=-\frac{\mathrm{d}^{2} u}{\mathrm{~d} y^{2}}$.

This mechanical model is now applied to elasto-plastic bending of the single crystal, both for loading and unloading. Taking $C_{i j}$ components from [26]: $C_{11}=206 \mathrm{GPa}$, $C_{12}=133 \mathrm{GPa}$ and $C_{44}=119 \mathrm{GPa}$, and using equation (2) we get $v=0.39$ and $E=102 \mathrm{GPa}$. The yield limit $\sigma_{0}$ and hardening modulus $H$ have been identified by least-square minimization between experimental and analytical elastic strain profiles, over all loadings steps, leading to $\sigma_{0}=62 \mathrm{GPa}$ and $H=13 \mathrm{GPa}$.

As seen in Figs. 7 and 8, elastic strain measurements successfully match with analytical profiles for all loadings. The mean distance between Laue-DIC results and predictions of the model is less than $2 \times 10^{-4}$.

The value of $\sigma_{0}$ agrees very well with the results of a tensile experiment along $<100>$ carried out on the same material by Chambreuil-Paret ( $\sim 60 \mathrm{GPa})$ [27]. The hardening modulus $H$ reflects the high hardening usually observed in the elasto-plastic transition of stress-strain tensile curves. In this experiment local total deformation in the sample is always below $0.5 \%$. Moreover, identified parameters give a macroscopic response of the model consistent with the experimental force-displacement curve (cf. Fig. 1).

Elastic strain fields measured by Laue-DIC in the deformed crystal are consistent with its known mechanical properties. Confident of the Laue-DIC results, we now 


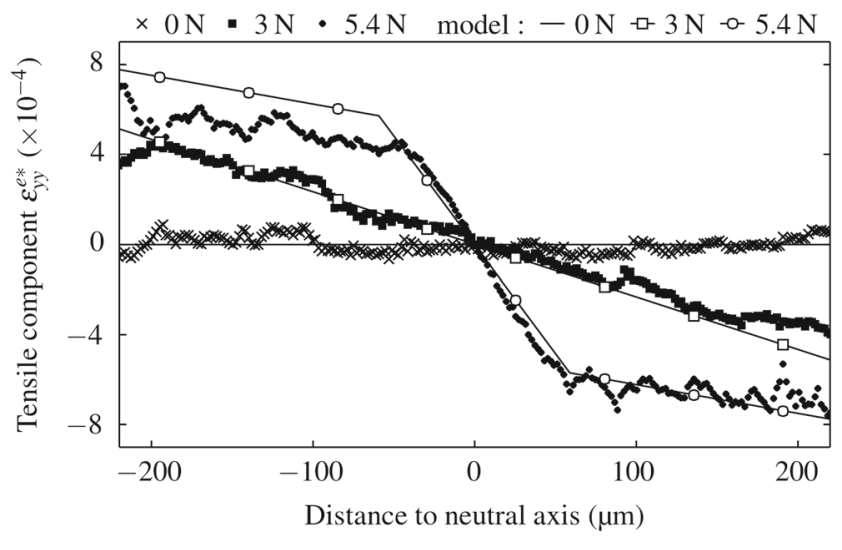

Fig. 7 Through-thickness elastic strain profiles measured by LaueDIC during in-situ bending on 3 loads. Comparison with the analytical model

take them as reference for cross-validation with HR-EBSD measurements.

\section{Cross-Validation with HR-EBSD}

General good agreement is found between the average profile from HR-EBSD and Laue-DIC (cf. Fig. 8). In the elastic part, the standard deviation of the distance between the two profiles for strain components is smaller than $10^{-4}$ : the values coincide within the level of dispersion of the HR-EBSD data.

In plastic regions the mean distance reaches $3.2 \times 10^{-4}$ (see Table 2). Some differences in trends certainly stem from the different depth of material probed by the two techniques $(\sim 40 \mu \mathrm{m}$ for synchrotron X-rays vs. $25 \mathrm{~nm}$ for backscattered electrons). Such a difference is not expected from a mechanical point of view as the uniaxial stress field, constant in a $y z$ plane, is compatible with the plane stress condition at the surface. Therefore the beam tilt or the penetration depth should have no influence on the measurements.

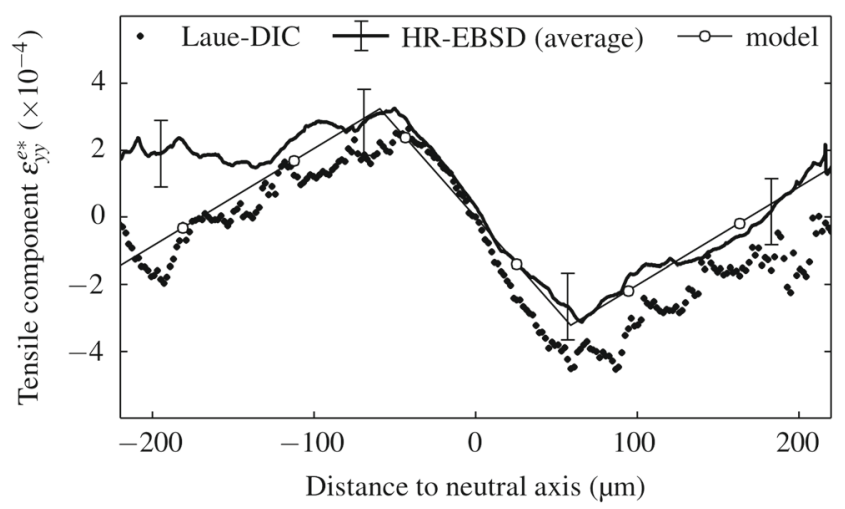

Fig. 8 Residual elastic strain profile measured by Laue-DIC and HREBSD after unloading (error bars give the standard deviation from averaged data). Comparison with the analytical model
However, the extremely local nature of EBSD measurements makes the data sensitive to local features like slip markings. Indeed a brief deviation from the general trend in HR-EBSD measurements are thought to match with specific rotation and strain states around single slip markings (not shown here).

With two independent techniques, a similar residual strain and rotation profile is measured. The results are consistent with the mechanical behavior of the single crystal. Therefore, we are confident of having measured the true strain values in the plastically deformed sample. An estimation of the accuracy (distance to the true value) can be drawn for the first time in plastically deformed sample. With this methodology, the accuracy of HR-EBSD and Laue-DIC is estimated at $3.2 \times 10^{-4}$.

\section{Conclusion}

This original experiment on a lightly deformed fcc crystal has been designed to validate measurements of local elastic strains in plastically deformed crystals by Laue-DIC and HR-EBSD. As a very good match between the two independant measurements has been found we can give the true values to within a range close to the dispersion of HREBSD data. Therefore our measurements in a steel sample deformed at plastic strains below $0.5 \%$ are accurate to $3.2 \times 10^{-4}$.

The experiment presented here was potentially sensitive to artefacts coming from experimental conditions. As plastic deformation level has not reached $0.5 \%$, artefacts due to the nature of plastic deformation were unlikely to impact strongly the measurements. However, the methodology introduced here (manufacturing a strain profile by four point bending in an alloy single crystal) is thought to be applied fruitfully to higher deformation levels, in order to tackle this last issue.

Acknowledgments This work has been financially supported by the French Research Agency ANR as part of the project MICROSTRESS (\#ANR-11-BS09-030). The synchrotron data used in this work was collected during ESRF experiment 32-02-748. We thank O. Geaymond for his help with setting up the Laue microdiffraction instrument. We gratefully acknowledge J. Stodolna, E. Bosso and J.-H. Driver for their advice.

\section{References}

1. Ice GE, Pang JW (2009) Tutorial on x-ray microlaue diffraction. Mater Charact 60(11):1191-1201. doi:10.1016/j.matchar.2009. 07.006

2. Wilkinson AJ, Meaden G, Dingley DJ (2006) High-resolution elastic strain measurement from electron backscatter diffraction patterns: New levels of sensitivity. Ultramicroscopy 106(45):307-313. doi:10.1016/j.ultramic.2005.10.001 
3. Petit J, Castelnau O, Bornert M, Zhang FG, Hofmann F, Korsunsky, AM, Faurie D, Le Bourlot C, Micha JS, Robach O, Ulrich O (2015) Laue-DIC: a new method for improved stress field measurements at the micrometer scale. J Synchrotron Radiat 22(4):980-994. doi:10.1107/S1600577515005780

4. Petit J, Bornert M, Hofmann F et al (2012) Combining laue microdiffraction and digital image correlation for improved measurements of the elastic strain field with micrometer spatial resolution. Procedia IUTAM 4(0):133-143. doi:10.1016/j.piutam.2012.05.015

5. Chung JS, Ice GE (1999) Automated indexing for texture and strain measurement with broad-bandpass x-ray microbeams. J Appl Phys 86(9):5249-5255. doi:10.1063/1.371507

6. Hofmann F, Eve S, Belnoue J, Micha JS, Korsunsky AM (2011) Analysis of strain error sources in micro-beam laue diffraction. Nuclear Instruments and Methods in Physics Research Section A: Accelerators, Spectrometers, Detectors and Associated Equipment 660(1):130-137. doi:10.1016/j.nima.2011.09.009

7. Villert S, Maurice C, Wyon C, Fortunier R (2009) Accuracy assessment of elastic strain measurement by EBSD. J Microsc 233(2):290-301. doi:10.1111/j.1365-2818.2009.03120.x

8. Poshadel A, Dawson P, Johnson G (2012) Assessment of deviatoric lattice strain uncertainty for polychromatic $\mathrm{x}$-ray microdiffraction experiments. J Synchrotron Radiat 19(2):237244

9. Magid K, Florando J, Lassila D, LeBlanc M, Tamura N, Morris J (2009) Mapping mesoscale heterogeneity in the plastic deformation of a copper single crystal. Phil Mag 89(1):77-107. doi:10.1080/14786430802558577

10. Jiang J, Britton TB, Wilkinson AJ (2013) Mapping type III intragranular residual stress distributions in deformed copper polycrystals. Acta Materialia 61(15):5895-5904

11. Larson BC, Levine LE (2013) Submicrometre-resolution polychromatic three-dimensional X-ray microscopy. J Appl Crystallogr 46(1):153-164

12. Maurice C, Driver JH, Fortunier R, On solving the orientation gradient dependency of high angular resolution EBSD (2012) Ultramicroscopy 113(0):171-181. doi:10.1016/j.ultramic.2011.10.013

13. Britton T, Wilkinson A (2012) High resolution electron backscatter diffraction measurements of elastic strain variations in the presence of larger lattice rotations. Ultramicroscopy 114(0):8295. doi:10.1016/j.ultramic.2012.01.004

14. Britton T, Maurice C, Fortunier R, Driver J, Day A, Meaden G, Dingley D, Mingard K, Wilkinson A (2010) Factors affecting the accuracy of high resolution electron backscatter diffraction when using simulated patterns. Ultramicroscopy 110(12):1443-1453. doi:10.1016/j.ultramic.2010.08.001

15. Maurice C, Dzieciol K, Fortunier R (2011) A method for accurate localisation of EBSD pattern centres. Ultramicroscopy 111(2):140-148. doi:10.1016/j.ultramic.2010.10.007

16. Mingard K, Day A, Maurice C, Quested P (2011) Towards high accuracy calibration of electron backscatter diffraction systems. Ultramicroscopy 111(5):320-329

17. Barabash RI, Ice GE, Liu W, Barabash OM (2009) Polychromatic microdiffraction characterization of defect gradients in severely deformed materials. Micron 40(1):28-36. doi:10.1016/j.micron.2008.03.010

18. Zhang FG, Castelnau O, Bornert M, Petit J, Marijon JB, Plancher E (2015) Determination of deviatoric elastic strain and lattice orientation by applying digital image correlation to Laue microdiffraction images - the enhanced Laue-DIC method. J Appl Crystallogr 48(6). doi:10.1107/S1600576715018397

19. Nolze G (2007) Image distortions in sem and their influences on ebsd measurements. Ultramicroscopy 107(2):172-183

20. Vaudin M, Stan G, Gerbig Y, Cook R (2011) High resolution surface morphology measurements using EBSD crosscorrelation techniques and AFM. Ultramicroscopy 111(8):12061213. doi:10.1016/j.ultramic.2011.01.039

21. Robach O, Kirchlechner C, Micha JS et al (2014) Strain and dislocation gradients from diffraction, chap. 5 : Laue microdiffraction at ESRF, pp 156-204. Imperial College Press / World Scientific Publishing

22. Ulrich $\mathrm{O}$, Biquard $\mathrm{X}$, Bleuet $\mathrm{P}$, Geaymond $\mathrm{O}$, Gergaud $\mathrm{P}$, Micha JS, Robach O, Rieutord F (2011) A new white beam $\mathrm{x}$-ray microdiffraction setup on the BM32 beamline at the european synchrotron radiation facility. Rev Sci Instrum 82(3). doi:10.1063/1.3555068

23. Bandyopadhyay P, Segre CU Mucal on the web. http://www.csrri. iit.edu/periodic-table.html

24. Drouin D, Couture AR, Joly D, Tastet X, Aimez V, Gauvin R (2007) Casino v2.42 a fast and easy-to-use modeling tool for scanning electron microscopy and microanalysis users. Scanning 29(3):92-101

25. Boehler JP, Sawczuk A (1977) On yielding of oriented solids. Acta Mech 27(1-4):185-204

26. Ledbetter H (1981) Predicted single-crystal elastic constants of stainless-steel 316. BR J NDT 23(6):286-287

27. Chambreuil-Paret A (1997) Ph.D. thesis, Ecole Nationale des Mines de Saint-Etienne / Institut Polytechnique de Grenoble (France). http://www.iaea.org/inis/collection/ NCLCollectionStore/\%_Public/29/040/29040011.pdf 